\title{
Stability of a Functional Differential System with a Finite Number of Delays
}

\author{
Josef Rebenda ${ }^{1}$ and Zdeněk Šmarda ${ }^{1,2}$ \\ ${ }^{1}$ CEITEC BUT, Brno University of Technology, Technicka 3058/10, 61600 Brno, Czech Republic \\ ${ }^{2}$ Department of Mathematics, Faculty of Electrical Engineering and Communication, Brno University of Technology, \\ Technicka 8, 61600 Brno, Czech Republic
}

Correspondence should be addressed to Zdeněk Šmarda; smarda@feec.vutbr.cz

Received 22 December 2012; Accepted 23 May 2013

Academic Editor: Zhiming Guo

Copyright (c) 2013 J. Rebenda and Z. Šmarda. This is an open access article distributed under the Creative Commons Attribution License, which permits unrestricted use, distribution, and reproduction in any medium, provided the original work is properly cited.

The paper is devoted to the study of asymptotic properties of a real two-dimensional differential system with unbounded nonconstant delays. The sufficient conditions for the stability and asymptotic stability of solutions are given. Used methods are based on the transformation of the considered real system to one equation with complex-valued coefficients. Asymptotic properties are studied by means of Lyapunov-Krasovskii functional. The results generalize some previous ones, where the asymptotic properties for two-dimensional systems with one or more constant delays or one nonconstant delay were studied.

\section{Introduction}

There are a lot of papers dealing with the stability and asymptotic behaviour of $n$-dimensional real vector equations with delay. Among others we should mention the recent results [1-13]. Since the plane has special topological properties different from those of $n$-dimensional space, where $n \geq 3$ or $n=1$, it is interesting to study asymptotic behaviour of two-dimensional systems by using tools which are typical and effective for two-dimensional systems. The convenient tool is the combination of the method of complexification and the method of Lyapunov-Krasovskii functional. Using these techniques we obtain new and easy applicable results on stability, asymptotic stability, or boundedness of solutions of real two-dimensional differential system

$$
\begin{aligned}
x^{\prime}(t)= & \mathrm{A}(t) x(t) \\
& +\sum_{k=1}^{m} \mathrm{~B}_{k}(t) x\left(\theta_{k}(t)\right) \\
& +h\left(t, x(t), x\left(\theta_{1}(t)\right), \ldots, x\left(\theta_{m}(t)\right)\right),
\end{aligned}
$$

where $\theta_{k}(t)$ are real functions, $\mathrm{A}(t)=\left(a_{i j}(t)\right), \mathrm{B}_{k}(t)=$ $\left(b_{i j k}(t)\right)(i, j=1,2 ; k=1, \ldots, m)$ are real square matrices, and $h(t, x, y)=\left(h_{1}\left(t, x, y_{1}, \ldots, y_{m}\right), h_{2}\left(t, x, y_{1}, \ldots, y_{m}\right)\right)$ is a real vector function, $x=\left(x_{1}, x_{2}\right), y_{k}=\left(y_{1 k}, y_{2 k}\right)$. It is supposed that the functions $\theta_{k}, a_{i j}$ are locally absolutely continuous on $\left[t_{0}, \infty\right), b_{i j k}$ are locally Lebesgue integrable on $\left[t_{0}, \infty\right)$, and the function $h$ satisfies Carathéodory conditions on $\left[t_{0}, \infty\right) \times$ $\mathbb{R}^{2(m+1)}$

Delayed differential equations recently gain more importance in applications in science and real world. They can be found in applications in medicine (control of drug therapies and neurological, physiological, and epidemiological models), biology (predator-prey models and blowflies lifecycle), chemistry (chemical kinetics), physics (private communication and signal masking), and engineering (machining operation on a lathe). Equation (1) represents a generalization of many of these models. Particularly, (1) in this general form has an application in modeling of multiple regenerative effect in tool chatter. Obtained results on stability give the possibility to find the best spindle speeds and depth-of-cut for the machines for chatter-free high-productivity operation. For more details, see [14].

The main idea of the investigation, the combination of the method of complexification and the method of LyapunovKrasovskii functional, was introduced for ordinary differential equations in the paper by Ráb and Kalas [15]. 
The principle was transferred to differential equations with delay by Kalas and Baráková [16]. The results for several constant delays can be found in papers by Rebenda [17, 18]. Differential equations with one nonconstant delay are studied by Kalas [19] and Rebenda [20].

We extend such type of results to differential equations with a finite number of nonconstant delays. We introduce the transformation of the considered real system to one equation with complex-valued coefficients. We present sufficient conditions for stability and asymptotic stability of a solution and the conditions under which all solutions tend to zero. The applicability of the results is demonstrated with an example.

At the end of this introduction we append an overview of notations used in the paper and the transformation of the real system to one equation with complex-valued coefficients.

Consider the following:

$\mathbb{R}$ : set of all real numbers,

$\mathbb{R}_{+}$: set of all positive real numbers,

$\mathbb{R}_{+}^{0}$ : set of all nonnegative real numbers,

$\mathbb{R}_{-}$: set of all negative real numbers,

$\mathbb{R}_{-}^{0}$ : set of all nonpositive real numbers,

$\mathbb{C}$ : set of all complex numbers,

$\mathscr{C}$ : class of all continuous functions $[-r, 0] \rightarrow \mathbb{C}$,

$A C_{\mathrm{loc}}(I, M)$ : class of all locally absolutely continuous functions $I \rightarrow M$,

$L_{\text {loc }}(I, M)$ : class of all locally Lebesgue integrable functions $I \rightarrow M$,

$K(I \times \Omega, M)$ : class of all functions $I \times \Omega \rightarrow M$ satisfying Carathéodory conditions on $I \times \Omega$,

$\operatorname{Re} z$ : real part of $z$,

$\operatorname{Im} z$ : imaginary part of $z$,

$\bar{z}$ : complex conjugate of $z$.

Introducing complex variables $z=x_{1}+i x_{2}, w_{1}=y_{11}+$ $i y_{12}, \ldots, w_{m}=y_{m 1}+i y_{m 2}$, we can rewrite system (1) into an equivalent equation with complex-valued coefficients:

$$
\begin{aligned}
z^{\prime}(t)= & a(t) z(t)+b(t) \bar{z}(t) \\
& +\sum_{k=1}^{m}\left[A_{k}(t) z\left(\theta_{k}(t)\right)+B_{k}(t) \bar{z}\left(\theta_{k}(t)\right)\right] \\
& +g\left(t, z(t), z\left(\theta_{1}(t)\right), \ldots, z\left(\theta_{m}(t)\right)\right),
\end{aligned}
$$

where $\theta_{k} \in A C_{\text {loc }}(J, \mathbb{R})$ for $k=1, \ldots, m, A_{k}, B_{k} \in L_{\text {loc }}(J, \mathbb{C})$, $a, b \in A C_{\mathrm{loc}}(J, \mathbb{C}), g \in K\left(J \times \mathbb{C}^{m+1}, \mathbb{C}\right), J=\left[t_{0}, \infty\right)$.
The relations between the functions are as follows:

$$
\begin{aligned}
& a(t)=\frac{1}{2}\left(a_{11}(t)+a_{22}(t)\right)+\frac{i}{2}\left(a_{21}(t)-a_{12}(t)\right), \\
& b(t)=\frac{1}{2}\left(a_{11}(t)-a_{22}(t)\right)+\frac{i}{2}\left(a_{21}(t)+a_{12}(t)\right), \\
& A_{k}(t)= \frac{1}{2}\left(b_{11 k}(t)+b_{22 k}(t)\right)+\frac{i}{2}\left(b_{21 k}(t)-b_{12 k}(t)\right), \\
& B_{k}(t)= \frac{1}{2}\left(b_{11 k}(t)-b_{22 k}(t)\right)+\frac{i}{2}\left(b_{21 k}(t)+b_{12 k}(t)\right), \\
& g\left(t, z, w_{1}, \ldots, w_{m}\right) h_{1}\left(t, \frac{1}{2}(z+\bar{z}), \frac{1}{2 i}(z-\bar{z}),\right. \\
&\left.\frac{1}{2}\left(w_{1}+\bar{w}_{1}\right), \ldots, \frac{1}{2 i}\left(w_{m}-\bar{w}_{m}\right)\right) \\
&+i h_{2}\left(t, \frac{1}{2}(z+\bar{z}), \frac{1}{2 i}(z-\bar{z}), \frac{1}{2}\left(w_{1}+\bar{w}_{1}\right)\right. \\
&\left.\frac{1}{2 i}\left(w_{1}-\bar{w}_{1}\right), \ldots, \frac{1}{2 i}\left(w_{m}-\bar{w}_{m}\right)\right) .
\end{aligned}
$$

Conversely, putting

$$
\begin{aligned}
& a_{11}(t)=\operatorname{Re}[a(t)+b(t)], \\
& a_{12}(t)=\operatorname{Im}[b(t)-a(t)], \\
& a_{21}(t)=\operatorname{Im}[a(t)+b(t)], \\
& a_{22}(t)=\operatorname{Re}[a(t)-b(t)], \\
& b_{11 k}(t)=\operatorname{Re}\left[A_{k}(t)+B_{k}(t)\right], \\
& b_{12 k}(t)=\operatorname{Im}\left[B_{k}(t)-A_{k}(t)\right], \\
& b_{21 k}(t)=\operatorname{Im}\left[A_{k}(t)+B_{k}(t)\right], \\
& b_{22 k}(t)=\operatorname{Re}\left[A_{k}(t)-B_{k}(t)\right], \\
& h_{1}\left(t, x, y_{1}, \ldots, y_{m}\right) \\
&=\operatorname{Re} g\left(t, x_{1}+i x_{2}, y_{11}+i y_{12}, \ldots, y_{m 1}+i y_{m 2}\right), \\
& h_{2}\left(t, x, y_{1}, \ldots, y_{m}\right) \\
&=\operatorname{Im} g\left(t, x_{1}+i x_{2}, y_{11}+i y_{12}, \ldots, y_{m 1}+i y_{m 2}\right),
\end{aligned}
$$

equation (2) can be written in real form (1) as well.

\section{Preliminaries}

We consider (2) in the case when

$$
\liminf _{t \rightarrow \infty}(|\operatorname{Im} a(t)|-|b(t)|)>0
$$

and study the behavior of solutions of (2) under this assumption. This situation corresponds to the case when the equilibrium 0 of the autonomous homogeneous system

$$
x^{\prime}=\mathbf{A} x
$$


where $\mathbf{A}$ is supposed to be regular constant matrix, is a centre or a focus.

This case is included in the case $\liminf _{t \rightarrow \infty}(|a(t)|-$ $|b(t)|)>0$ considered in [21], but in this special case, we are able to derive more useful results as we will see later in an example. The idea is based on the well-known result that the condition $|a|>|b|$ in an autonomous equation $z^{\prime}=a z+b \bar{z}$ ensures that zero is a focus, a centre, or a node while under the condition $|\operatorname{Im} a|>|b|$ zero can be just a focus or a centre. Details are found in [15].

A simple example shows that, in some cases, the results of this paper can be applied more suitably than those given in [21].

Regarding (5) and since the delay functions $\theta_{k}$ satisfy $\lim _{t \rightarrow \infty} \theta_{k}(t)=\infty$, there are numbers $T_{1} \geq t_{0}, T \geq T_{1}$, and $\mu>0$ such that

$$
\begin{gathered}
\quad|\operatorname{Im} a(t)|>|b(t)|+\mu \quad \text { for } t \geq T_{1}, \\
t \geq \theta_{k}(t) \geq T_{1} \quad \text { for } t \geq T \quad(k=1, \ldots, m) .
\end{gathered}
$$

Denote

$$
\begin{gathered}
\tilde{\gamma}(t)=\operatorname{Im} a(t)+\sqrt{(\operatorname{Im} a(t))^{2}-|b(t)|^{2}} \operatorname{sgn}(\operatorname{Im} a(t)), \\
\widetilde{c}(t)=-i b(t) .
\end{gathered}
$$

Notice that, unlike the function $\gamma$ introduced in [21], the previously defined function $\tilde{\gamma}$ need not be positive.

Since $|\widetilde{\gamma}(t)|>|\operatorname{Im} a(t)|$ and $|\widetilde{c}(t)|=|b(t)|$, the inequality

$$
|\widetilde{\gamma}(t)|>|\widetilde{c}(t)|+\mu
$$

is valid for $t \geq T_{1}$. It can be easily verified that $\tilde{\gamma}, \widetilde{c} \in$ $A C_{\text {loc }}\left(\left[T_{1}, \infty\right), \mathbb{C}\right)$.

For the rest of this section, denote that

$$
\begin{aligned}
& \widetilde{\vartheta}(t) \\
& =\frac{\operatorname{Re}\left(\tilde{\gamma}(t) \tilde{\gamma}^{\prime}(t)-\overline{\widetilde{c}}(t) \tilde{c}^{\prime}(t)\right)-\left|\widetilde{\gamma}(t) \tilde{c}^{\prime}(t)-\tilde{\gamma}^{\prime}(t) \widetilde{c}(t)\right|}{\tilde{\gamma}^{2}(t)-|\widetilde{c}(t)|^{2}} .
\end{aligned}
$$

The stability and asymptotic stability are studied under the following assumptions.

(i) The numbers $T_{1} \geq t_{0}, T \geq T_{1}$, and $\mu>0$ are such that (7) holds.

(ii) There exist functions $\tilde{\mathcal{\varkappa}}, \widetilde{\kappa}_{k}, \widetilde{\varrho}:[T, \infty) \rightarrow \mathbb{R}$ such that

$$
\begin{aligned}
& \mid \widetilde{\gamma}(t) g\left(t, z, w_{1}, \ldots, w_{m}\right) \\
&+\widetilde{c}(t) \bar{g}\left(t, z, w_{1}, \ldots, w_{m}\right) \mid \\
& \leq \tilde{\mathcal{H}}(t)|\widetilde{\gamma}(t) z+\widetilde{c}(t) \bar{z}| \\
&+\sum_{k=1}^{m} \widetilde{\kappa}_{k}(t) \mid \widetilde{\gamma}\left(\theta_{k}(t)\right) w_{k} \\
&+\widetilde{\mathcal{c}}\left(\theta_{k}(t)\right) \bar{w}_{k} \mid+\widetilde{\varrho}(t),
\end{aligned}
$$

for $t \geq T, z, w_{k} \in \mathbb{C}(k=1, \ldots, m)$, where $\tilde{\mathcal{x}}, \widetilde{\varrho} \in$ $L_{\text {loc }}([T, \infty), \mathbb{R})$.

(iii) $\widetilde{\beta} \in A C_{\text {loc }}\left([T, \infty), \mathbb{R}_{+}^{0}\right)$ is a function satisfying

$$
\theta_{k}^{\prime}(t) \widetilde{\beta}(t) \geq \widetilde{\lambda}_{k}(t) \quad \text { a.e. on }[T, \infty),
$$

where $\tilde{\lambda}_{k}$ is defined for $t \geq T$ by

$$
\begin{aligned}
\widetilde{\lambda}_{k}(t)= & \widetilde{\kappa}_{k}(t)+\left(\left|A_{k}(t)\right|+\left|B_{k}(t)\right|\right) \\
& \times \frac{|\widetilde{\gamma}(t)|+|\widetilde{c}(t)|}{\left|\widetilde{\gamma}\left(\theta_{k}(t)\right)\right|-\left|\widetilde{c}\left(\theta_{k}(t)\right)\right|} .
\end{aligned}
$$

(iv) There exists a function $\widetilde{\Lambda} \in L_{\text {loc }}([T, \infty), \mathbb{R})$ which satisfies the inequalities $\widetilde{\beta}^{\prime}(t) \leq \widetilde{\Lambda}(t) \widetilde{\beta}(t), \widetilde{\Theta}(t) \leq \widetilde{\Lambda}(t)$ for almost all $t \in[T, \infty)$, where the function $\widetilde{\Theta}$ is defined by

$$
\widetilde{\Theta}(t)=\operatorname{Re} a(t)+\widetilde{\vartheta}(t)+\tilde{\varkappa}(t)+m \widetilde{\beta}(t) .
$$

If $A_{k}, B_{k}, \widetilde{\kappa}_{k}, \theta_{k}^{\prime}$ are locally absolutely continuous on $[T, \infty)$ and $\tilde{\lambda}_{k}(t) \geq 0, \theta_{k}^{\prime}(t)>0$ on $[T, \infty)$, the choice $\widetilde{\beta}(t)=$ $\max _{k=1, \ldots, m}\left[\tilde{\lambda}_{k}(t)\left(\theta_{k}^{\prime}(t)\right)^{-1}\right]$ is admissible in (iii).

From the assumption (i), it follows that

$$
\begin{aligned}
|\widetilde{\vartheta}| & \leq \frac{\left|\operatorname{Re}\left(\tilde{\gamma} \widetilde{\gamma}^{\prime}-\overline{\bar{c} c^{\prime}}\right)\right|+\left|\widetilde{\gamma} \widetilde{c}^{\prime}-\widetilde{\gamma}^{\prime} \tilde{c}\right|}{\widetilde{\gamma}^{2}-|\widetilde{c}|^{2}} \\
& \leq \frac{\left(\left|\tilde{\gamma}^{\prime}\right|+\left|\widetilde{c}^{\prime}\right|\right)(|\widetilde{\gamma}|+|\widetilde{c}|)}{\widetilde{\gamma}^{2}-|\widetilde{c}|^{2}} \\
& =\frac{\left|\widetilde{\gamma}^{\prime}\right|+\left|\widetilde{c}^{\prime}\right|}{|\widetilde{\gamma}|-|\widetilde{c}|} \leq \frac{1}{\mu}\left(\left|\tilde{\gamma}^{\prime}\right|+\left|\widetilde{c}^{\prime}\right|\right) ;
\end{aligned}
$$

hence, the function $\widetilde{\vartheta}$ is locally Lebesgue integrable on $[T, \infty)$. Moreover, if $\widetilde{\beta} \in A C_{\mathrm{loc}}\left([T, \infty), \mathbb{R}_{+}\right)$and $\tilde{\varkappa} \in L_{\mathrm{loc}}([T, \infty)$, then we can choose

$$
\widetilde{\Lambda}(t)=\max \left(\widetilde{\Theta}(t), \frac{\widetilde{\beta}^{\prime}(t)}{\widetilde{\beta}(t)}\right),
$$

in (iv).

Finally, if $\widetilde{\varrho}(t) \equiv 0$ in (ii), then (2) has the trivial solution $z(t) \equiv 0$. Notice that in this case the condition (ii) implies that the functions $\tilde{\varkappa}(t), \widetilde{\kappa}_{k}(t)$ are nonnegative on $[T, \infty)$ for $k=1, \ldots, m$, and due to this, $\tilde{\lambda}_{k}(t) \geq 0$ on $[T, \infty)$. The case $\widetilde{\varrho}(t)<0$ is omitted since it can be replaced by $\widetilde{\varrho}(t) \equiv 0$.

\section{Main Results}

The aim is to generalize the results for ordinary differential equations published in [15] as well as the results contained in [16] (one constant delay), [18] (a finite number of constant delays), and [20] (one nonconstant delay). In the proof of the crucial theorem, we use the following auxiliary result. 
Lemma 1. Let $a_{1}, a_{2}, b_{1}, b_{2} \in \mathbb{C}$ and $\left|a_{2}\right|>\left|b_{2}\right|$. Then

$$
\operatorname{Re} \frac{a_{1} z+b_{1} \bar{z}}{a_{2} z+b_{2} \bar{z}} \leq \frac{\operatorname{Re}\left(a_{1} \bar{a}_{2}-b_{1} \bar{b}_{2}\right)+\left|a_{1} b_{2}-a_{2} b_{1}\right|}{\left|a_{2}\right|^{2}-\left|b_{2}\right|^{2}},
$$

for $z \in \mathbb{C}, z \neq 0$.

The proof of Lemma 1 can be found, for example, in [15, page 131] or [17, page 101].

Theorem 2. Let the conditions (i), (ii), (iii), and (iv) hold and $\widetilde{\varrho}(t) \equiv 0$.

(a) If

$$
\limsup _{t \rightarrow \infty} \int^{t} \widetilde{\Lambda}(s) d s<\infty,
$$

then the trivial solution of (2) is stable on $[T, \infty)$.

(b) If

$$
\lim _{t \rightarrow \infty} \int^{t} \tilde{\Lambda}(s) d s=-\infty
$$

then the trivial solution of (2) is asymptotically stable on $[T, \infty)$.

Proof. Choose arbitrary $t_{1} \geq T$. Let $z(t)$ be any solution of (2) satisfying the condition $z(t)=z_{0}(t)$ for $t \in\left[T_{1}, t_{1}\right]$, where $z_{0}(t)$ is a continuous complex-valued initial function defined on $t \in\left[T_{1}, t_{1}\right]$. Consider the Lyapunov functional

$$
V(t)=U(t)+\widetilde{\beta}(t) \sum_{k=1}^{m} \int_{\theta_{k}(t)}^{t} U(s) d s,
$$

where

$$
U(t)=|\widetilde{\gamma}(t) z(t)+\widetilde{c}(t) \bar{z}(t)| .
$$

To simplify the computations, denote that $w_{k}(t)=$ $z\left(\theta_{k}(t)\right)$ and write the functions of variable $t$ without brackets, for example, $z$ instead of $z(t)$.

From (20) we get

$$
\begin{aligned}
V^{\prime}= & U^{\prime}+\widetilde{\beta}^{\prime} \sum_{k=1}^{m} \int_{\theta_{k}(t)}^{t} U(s) d s+m \tilde{\beta}|\tilde{\gamma} z+\widetilde{c} \bar{z}| \\
& -\sum_{k=1}^{m} \theta_{k}^{\prime} \widetilde{\beta}\left|\widetilde{\gamma}\left(\theta_{k}\right) w_{k}+\widetilde{c}\left(\theta_{k}\right) \bar{w}_{k}\right|,
\end{aligned}
$$

for almost all $t \geq t_{1}$ for which $z(t)$ is defined and $U^{\prime}(t)$ exists.

Denote that $\mathscr{K}=\left\{t \geq t_{1}: z(t)\right.$ exists, $\left.U(t) \neq 0\right\}$ and $\mathscr{M}=$ $\left\{t \geq t_{1}: z(t)\right.$ exists, $\left.U(t)=0\right\}$. It is clear that the derivative $U^{\prime}(t)$ exists for almost all $t \in \mathscr{K}$; hence, we focus on the set $M$.
In view of (9) we have $z(t)=0$ for $t \in \mathscr{M}$. For almost all $t \in \mathscr{M}$, we compute

$$
\begin{aligned}
U_{ \pm}^{\prime}(t) & =\lim _{\tau \rightarrow t \pm} \frac{U(\tau)-U(t)}{\tau-t} \\
& =\lim _{\tau \rightarrow t \pm} \frac{U(\tau)}{\tau-t} \\
& =\lim _{\tau \rightarrow t \pm} \frac{|\widetilde{\gamma}(\tau)[z(\tau)-z(t)]-\widetilde{c}(\tau)[\bar{z}(\tau)-\bar{z}(t)]|}{\tau-t} \\
& = \pm\left|\widetilde{\gamma}(t) z^{\prime}(t)+\widetilde{c}(t) \bar{z}^{\prime}(t)\right| \\
& = \pm\left|\widetilde{\gamma}(t) g^{*}(t)+\widetilde{c}(t) \bar{g}^{*}(t)\right|,
\end{aligned}
$$

where

$$
\begin{aligned}
g^{*}(t)= & \sum_{k=1}^{m}\left(A_{k}(t) w_{k}(t)+B_{k}(t) \bar{w}_{k}(t)\right) \\
& +g\left(t, 0, w_{1}(t), \ldots, w_{m}(t)\right) .
\end{aligned}
$$

Hence, $U$ has one-sided derivatives a.e. in $\mathscr{M}$. According to [22, Chapter IX., Theorem (1.1)] or [23], the set of all $t$ such that $U_{+}^{\prime}(t) \neq U_{-}^{\prime}(t)$ can be at most countable; thus, the derivative $U^{\prime}$ exists for almost all $t \in \mathscr{M}$, and for these $t$, $U^{\prime}(t)=0$.

In particular, the derivative $U^{\prime}$ exists for almost all $t \geq t_{1}$ for which $z(t)$ is defined; thus, (22) holds for almost all $t \geq t_{1}$ for which $z(t)$ is defined.

Now return the attention to the set $\mathscr{K}$. For almost all $t \in$ $\mathscr{K}$, it holds that $U U^{\prime}=U\left(\sqrt{(\tilde{\gamma} z+\tilde{c} \bar{z})(\overline{\tilde{\gamma}} \bar{z}+\overline{\tilde{c}} z)}^{\prime}=\operatorname{Re}[(\tilde{\gamma} \bar{z}+\right.$ $\left.\overline{\tilde{c}} z)\left(\tilde{\gamma}^{\prime} z+\tilde{\gamma} z^{\prime}+\tilde{c}^{\prime} \bar{z}+\widetilde{c} \bar{z}^{\prime}\right)\right]$. As $z(t)$ is a solution of (2), we have

$$
\begin{aligned}
& U U^{\prime}=\operatorname{Re}\{(\tilde{\gamma} \bar{z}+\overline{\tilde{c}} z) \\
& \times\left[\tilde{\gamma}^{\prime} z+\tilde{c}^{\prime} \bar{z}\right. \\
& +\widetilde{\gamma}\left(a z+b \bar{z}+\sum_{k=1}^{m}\left(A_{k} w_{k}+B_{k} \bar{w}_{k}\right)+g\right) \\
& \left.\left.+\widetilde{c}\left(\bar{a} \bar{z}+\bar{b} z+\sum_{k=1}^{m}\left(\bar{A}_{k} \bar{w}_{k}+\bar{B}_{k} w_{k}\right)+\bar{g}\right)\right]\right\} \\
& =\operatorname{Re}\{(\tilde{\gamma} z+\overline{\tilde{c}} z) \\
& \times\left[\widetilde{\gamma}^{\prime} z+\widetilde{c}^{\prime} \bar{z}+(\tilde{\gamma} a+\bar{c} \bar{b}) z\right. \\
& +(\tilde{\gamma} b+\widetilde{c} a) \bar{z}
\end{aligned}
$$




$$
\begin{aligned}
& +\widetilde{\gamma}\left(\sum_{k=1}^{m}\left(A_{k} w_{k}+B_{k} \bar{w}_{k}\right)+g\right) \\
& \left.\left.+\widetilde{c}\left(\sum_{k=1}^{m}\left(\bar{A}_{k} \bar{w}_{k}+\bar{B}_{k} w_{k}\right)+\bar{g}\right)\right]\right\},
\end{aligned}
$$

for almost all $t \in \mathscr{K}$.

Short computation gives $(\widetilde{\gamma} a+\bar{c} \bar{b}) \widetilde{c}=(\widetilde{\gamma} b+\widetilde{c} \bar{a}) \widetilde{\gamma}$, and from this we get

$$
\begin{aligned}
& U U^{\prime} \leq \operatorname{Re}\left\{(\tilde{\gamma} \bar{z}+\overline{\widetilde{c}} z)(\tilde{\gamma} a+\bar{c} \bar{b})\left(z+\frac{\tilde{c}}{\widetilde{\gamma}} \bar{z}\right)\right\} \\
& +\operatorname{Re}\left\{( \tilde { \gamma } \overline { z } + \overline { \tilde { c } } z ) \left[\tilde{\gamma} \sum_{k=1}^{m}\left(A_{k} w_{k}+B_{k} \bar{w}_{k}\right)\right.\right. \\
& \left.\left.+\widetilde{c} \sum_{k=1}^{m}\left(\bar{A}_{k} \bar{w}_{k}+\bar{B}_{k} w_{k}\right)\right]\right\} \\
& +\operatorname{Re}[(\widetilde{\gamma} z+\overline{\widetilde{c}} z)(\tilde{\gamma} g+\widetilde{c} g)] \\
& +\operatorname{Re}\left[(\widetilde{\gamma} z+\overline{\widetilde{c}} z)\left(\widetilde{\gamma}^{\prime} z+\widetilde{c}^{\prime} \bar{z}\right)\right] \\
& \leq U^{2} \operatorname{Re}\left(a+\frac{\widetilde{c}}{\tilde{\gamma}} \bar{b}\right) \\
& +U(|\widetilde{\gamma}|+|\widetilde{c}|)\left(\sum_{k=1}^{m}\left|A_{k} w_{k}+B_{k} \bar{w}_{k}\right|\right) \\
& +U|\tilde{\gamma} g+\widetilde{c} g|+U^{2} \operatorname{Re} \frac{\tilde{\gamma}^{\prime} z+\tilde{c}^{\prime} \bar{z}}{\tilde{\gamma} z+\widetilde{c} z},
\end{aligned}
$$

for almost all $t \in \mathscr{K}$.

Applying Lemma 1 to the last term, we obtain

$$
\operatorname{Re} \frac{\tilde{\gamma}^{\prime} z+\tilde{c}^{\prime} \bar{z}}{\tilde{\gamma} z+\widetilde{c} z} \leq \widetilde{\vartheta}
$$

Using this inequality together with (13), assumption (ii), and the relation $\operatorname{Re}(a+(\widetilde{c} / \widetilde{\gamma}) \bar{b})=\operatorname{Re} a$, we obtain

$$
\begin{aligned}
U U^{\prime} \leq & U^{2}(\operatorname{Re} a+\widetilde{\vartheta}+\tilde{\varkappa}) \\
& +U \sum_{k=1}^{m}\left(\widetilde{\kappa}_{k}\left|\widetilde{\gamma}\left(\theta_{k}\right) w_{k}+\widetilde{c}\left(\theta_{k}\right) \bar{w}_{k}\right|\right) \\
& +U(|\widetilde{\gamma}|+|\widetilde{c}|) \\
& \times\left(\sum_{k=1}^{m} \frac{\left|A_{k}\right|\left|w_{k}\right|+\left|B_{k}\right|\left|\bar{w}_{k}\right|}{\left|\widetilde{\gamma}\left(\theta_{k}\right)\right|-\left|\widetilde{c}\left(\theta_{k}\right)\right|}\left(\left|\widetilde{\gamma}\left(\theta_{k}\right)\right|-\left|\widetilde{c}\left(\theta_{k}\right)\right|\right)\right) \\
\leq & U^{2}(\operatorname{Re} a+\widetilde{\vartheta}+\tilde{\varkappa})
\end{aligned}
$$

$$
\begin{aligned}
& +U\left\{\sum_{k=1}^{m}\left[\widetilde{\kappa}_{k}+\left(\left|A_{k}\right|+\left|B_{k}\right|\right) \frac{|\widetilde{\gamma}|+|\tilde{c}|}{\left|\widetilde{\gamma}\left(\theta_{k}\right)\right|-\left|\widetilde{c}\left(\theta_{k}\right)\right|}\right]\right. \\
& \left.\quad \times\left|\widetilde{\gamma}\left(\theta_{k}\right) w_{k}+\widetilde{c}\left(\theta_{k}\right) \bar{w}_{k}\right|\right\} \\
& \leq U^{2}(\operatorname{Re} a+\widetilde{\vartheta}+\tilde{\varkappa}) \\
& +U \sum_{k=1}^{m} \widetilde{\lambda}_{k}\left|\widetilde{\gamma}\left(\theta_{k}\right) w_{k}+\widetilde{c}\left(\theta_{k}\right) \bar{w}_{k}\right|,
\end{aligned}
$$

for almost all $t \in \mathscr{K}$.

Consequently,

$$
U^{\prime} \leq U(\operatorname{Re} a+\widetilde{\vartheta}+\tilde{\varkappa})+\sum_{k=1}^{m} \widetilde{\lambda}_{k}\left|\widetilde{\gamma}\left(\theta_{k}\right) w_{k}+\widetilde{c}\left(\theta_{k}\right) \bar{w}_{k}\right|,
$$

for almost all $t \in \mathscr{K}$.

Recalling that $U^{\prime}(t)=0$ for almost all $t \in \mathscr{M}$, we can see that inequality (29) is valid for almost all $t \geq t_{1}$ for which $z(t)$ is defined.

From (29) we have

$$
\begin{aligned}
V^{\prime} \leq & U(\operatorname{Re} a+\widetilde{\vartheta}+\tilde{\varkappa}+m \tilde{\beta}) \\
& +\sum_{k=1}^{m}\left(\tilde{\lambda}_{k}-\theta_{k}^{\prime} \widetilde{\beta}\right)\left|\widetilde{\gamma}\left(\theta_{k}\right) w_{k}+\widetilde{c}\left(\theta_{k}\right) \bar{w}_{k}\right| \\
& +\widetilde{\beta}^{\prime} \sum_{k=1}^{m} \int_{\theta_{k}(t)}^{t}|\widetilde{\gamma}(s) z(s)+\widetilde{c}(s) \bar{z}(s)| d s .
\end{aligned}
$$

As $\widetilde{\beta}(t)$ fulfills condition (12), we obtain

$$
\begin{aligned}
V^{\prime}(t) \leq & U(t) \widetilde{\Theta}(t) \\
& +\widetilde{\beta}^{\prime}(t) \sum_{k=1}^{m} \int_{\theta_{k}(t)}^{t}|\widetilde{\gamma}(s) z(s)+\widetilde{c}(s) \bar{z}(s)| d s,
\end{aligned}
$$

Hence,

$$
V^{\prime}(t)-\widetilde{\Lambda}(t) V(t) \leq 0,
$$

for almost all $t \geq t_{1}$ for which the solution $z(t)$ exists.

Notice that, with respect to (9),

$$
V(t) \geq(|\widetilde{\gamma}(t)|-|\widetilde{c}(t)|)|z(t)| \geq \mu|z(t)|,
$$

for all $t \geq t_{1}$ for which $z(t)$ is defined.

Suppose that condition (18) holds, and choose arbitrary $\varepsilon>0$. Put

$$
\begin{gathered}
\Delta=\max _{s \in\left[T_{1}, t_{1}\right]}(|\tilde{\gamma}(s)|+|\widetilde{c}(s)|), \quad L=\sup _{T \leq t<\infty} \int_{T}^{t} \widetilde{\Lambda}(s) d s, \\
\delta=\mu \varepsilon \Delta^{-1}\left(1+m \widetilde{\beta}\left(t_{1}\right)\left(t_{1}-T_{1}\right)\right)^{-1} \exp \left\{\int_{T}^{t_{1}} \widetilde{\Lambda}(s) d s-L\right\},
\end{gathered}
$$


where $\mu>0, T_{1} \geq t_{0}$, and $T \geq T_{1}$ are the numbers from condition (i).

If the initial function $z_{0}(t)$ of the solution $z(t)$ satisfies $\max _{s \in\left[T_{1}, t_{1}\right]}\left|z_{0}(s)\right|<\delta$, then the multiplication of (32) by $\exp \left\{-\int_{t_{1}}^{t} \widetilde{\Lambda}(s) d s\right\}$ and the integration over $\left[t_{1}, t\right]$ yield

$$
V(t) \exp \left\{-\int_{t_{1}}^{t} \tilde{\Lambda}(s) d s\right\}-V\left(t_{1}\right) \leq 0,
$$

for all $t \geq t_{1}$ for which $z(t)$ is defined. From (33) and (35) we get

$$
\begin{aligned}
& \mu|z(t)| \leq V(t) \leq V\left(t_{1}\right) \exp \left\{\int_{t_{1}}^{t} \widetilde{\Lambda}(s) d s\right\} \\
& \leq\left[\left(\left|\widetilde{\gamma}\left(t_{1}\right)\right|+\left|\widetilde{c}\left(t_{1}\right)\right|\right)\left|z\left(t_{1}\right)\right|\right. \\
& +\widetilde{\beta}\left(t_{1}\right) \max _{s \in\left[T_{1}, t_{1}\right]}|z(s)| \\
& \left.\times\left(\sum_{k=1}^{m} \int_{\theta_{k}\left(t_{1}\right)}^{t_{1}}(|\widetilde{\gamma}(s)|+|\widetilde{\mathcal{c}}(s)|) d s\right)\right] \\
& \times \exp \left\{\int_{t_{1}}^{t} \widetilde{\Lambda}(s) d s\right\} \\
& \leq\left[\Delta \max _{s \in\left[T_{1}, t_{1}\right]}\left|z_{0}(s)\right|\right. \\
& +\widetilde{\beta}\left(t_{1}\right) \max _{s \in\left[T_{1}, t_{1}\right]}\left|z_{0}(s)\right| \Delta \\
& \left.\times \sum_{k=1}^{m}\left(t_{1}-\theta_{k}\left(t_{1}\right)\right)\right] \\
& \times \exp \left\{\int_{t_{1}}^{t} \widetilde{\Lambda}(s) d s\right\} ;
\end{aligned}
$$

that is,

$$
\begin{aligned}
\mu|z(t)| \leq & \Delta \max _{s \in\left[T_{1}, t_{1}\right]}\left|z_{0}(s)\right|\left(1+m \tilde{\beta}\left(t_{1}\right)\left(t_{1}-T_{1}\right)\right) \\
& \times \exp \left\{L-\int_{T}^{t_{1}} \widetilde{\Lambda}(s) d s\right\}<\mu \varepsilon .
\end{aligned}
$$

Thus, we have $|z(t)|<\varepsilon$ for all $t \geq t_{1}$, and we conclude that the trivial solution of (2) is stable.

Now suppose that condition (19) is valid. Then, in view of the first part of Theorem 2 , for $K>0$, there is a $\rho>0$ such that $\max _{s \in\left[T_{1}, t_{1}\right]}\left|z_{0}(s)\right|<\rho$ implies that the solution $z(t)$ of (2) exists for all $t \geq t_{1}$ and satisfies $|z(t)|<K$, where $K$ is arbitrary real constant. Hence, from this and (33), we have

$$
|z(t)| \leq \mu^{-1} V(t) \leq \mu^{-1} V\left(t_{1}\right) \exp \left\{\int_{t_{1}}^{t} \widetilde{\Lambda}(s) d s\right\},
$$

for all $t \geq t_{1}$. This inequality, with condition (19), gives

$$
\lim _{t \rightarrow \infty} z(t)=0,
$$

which completes the proof.
Remark 3. Theorem 2 represents a generalization of previous results.

If we take $A_{1}(t)=A(t), A_{k} \equiv 0$, for $k=2, \ldots, m, B_{1}(t)=$ $B(t), B_{k} \equiv 0$, for $k=2, \ldots, m$, and $\theta_{1}(t)=t-r$, where $r>0$, we get Theorem 4 from [16].

If we take $\theta_{k}(t)=t-r_{k}$, where $r_{k}>0, k=1, \ldots, m$, we obtain Theorem 1 from [18].

If we take $A_{1}(t)=A(t), A_{k} \equiv 0$, for $k=2, \ldots, m$, $B_{1}(t)=B(t), B_{k} \equiv 0$, for $k=2, \ldots, m$, and $\theta_{1}(t)=\theta(t)$, we get Theorem 2.2 from [20].

The next theorem involves the function $\widetilde{\varrho}$ in (ii); thus, it is more general than Theorem 2. A part of the proof of Theorem 2 is utilized in the proof of Theorem 4 .

Theorem 4. Let the assumptions (i), (ii), (iii), and (iv) hold and

$$
\begin{aligned}
V(t)= & |\widetilde{\gamma}(t) z(t)+\widetilde{c}(t) \bar{z}(t)| \\
& +\widetilde{\beta}(t) \sum_{k=1}^{m} \int_{\theta_{k}(t)}^{t}|\widetilde{\gamma}(s) z(s)+\widetilde{c}(s) \bar{z}(s)| d s,
\end{aligned}
$$

where $z(t)$ is any solution of $(2)$ defined for $t \rightarrow \infty$. Then

$$
\begin{aligned}
\mu|z(t)| \leq & V(s) \exp \left(\int_{s}^{t} \widetilde{\Lambda}(\xi) d \xi\right) \\
& +\int_{s}^{t} \widetilde{\varrho}(\xi) \exp \left(\int_{\xi}^{t} \widetilde{\Lambda}(\sigma) d \sigma\right) d \xi,
\end{aligned}
$$

for $t \geq s \geq t_{1}$, where $t_{1} \geq T$.

Proof. Following the proof of Theorem 2, we have

$$
\begin{aligned}
V^{\prime}(t) \leq & |\widetilde{\gamma}(t) z(t)+\widetilde{c}(t) \bar{z}(t)| \Theta(t) \\
& +\widetilde{\beta}^{\prime}(t) \sum_{k=1}^{m} \int_{\theta_{k}(t)}^{t}|\widetilde{\gamma}(s) z(s)+\widetilde{c}(s) \bar{z}(s)| d s \\
& +\widetilde{\varrho}(t) \\
\leq & \widetilde{\Lambda}(t) V(t)+\widetilde{\varrho}(t),
\end{aligned}
$$

a.e. on $\left[t_{1}, \infty\right)$. Using this inequality, we get

$$
V^{\prime}(t)-\widetilde{\Lambda}(t) V(t) \leq \widetilde{\varrho}(t),
$$

a.e. on $\left[t_{1}, \infty\right)$. Multiplying (43) by $\exp \left(-\int_{s}^{t} \widetilde{\Lambda}(\xi) d \xi\right)$ gives

$$
\left[V(t) \exp \left(-\int_{s}^{t} \widetilde{\Lambda}(\xi) d \xi\right)\right] \leq \widetilde{\varrho}(t) \exp \left(-\int_{s}^{t} \widetilde{\Lambda}(\xi) d \xi\right),
$$

a.e. on $\left[t_{1}, \infty\right)$. Integration over $[s, t]$ yields

$$
\begin{aligned}
V(t) & \exp \left(-\int_{s}^{t} \widetilde{\Lambda}(\xi) d \xi\right)-V(s) \\
& \leq \int_{s}^{t} \widetilde{\varrho}(\xi) \exp \left(-\int_{s}^{\xi} \widetilde{\Lambda}(\sigma) d \sigma\right) d \xi,
\end{aligned}
$$


and multiplying (45) by $\exp \left(\int_{s}^{t} \widetilde{\Lambda}(\xi) d \xi\right)$, we obtain

$$
\begin{aligned}
V(t) \leq & V(s) \exp \left(\int_{s}^{t} \widetilde{\Lambda}(\xi) d \xi\right) \\
& +\int_{s}^{t} \widetilde{\varrho}(\xi) \exp \left(\int_{\xi}^{t} \widetilde{\Lambda}(\sigma) d \sigma\right) d \xi .
\end{aligned}
$$

The statement now follows from (33).

Remark 5. Theorem 4 generalizes theorems contained in previous papers.

If we take $A_{1}(t)=A(t), A_{k} \equiv 0$, for $k=2, \ldots, m, B_{1}(t)=$ $B(t), B_{k} \equiv 0$, for $k=2, \ldots, m$, and $\theta_{1}(t)=t-r$, where $r>0$, we get Theorem 2 from [16].

If we take $\theta_{k}(t)=t-r_{k}$, where $r_{k}>0, k=1, \ldots, m$, we obtain Theorem 2 from [18].

If we take $A_{1}(t)=A(t), A_{k} \equiv 0$, for $k=2, \ldots, m$, $B_{1}(t)=B(t), B_{k} \equiv 0$, for $k=2, \ldots, m$, and $\theta_{1}(t)=\theta(t)$, we get Theorem 2.7 from [20].

The last of the main propositions gives the conditions under which all solutions of (2) tend to zero.

Theorem 6. Let the assumptions (i), (ii), (iii), and (iv) be satisfied. Let $\widetilde{\Lambda}(t) \leq 0$ a.e. on $\left[T^{*}, \infty\right)$, where $T^{*} \in[T, \infty)$. If

$$
\lim _{t \rightarrow \infty} \int^{t} \widetilde{\Lambda}(s) d s=-\infty, \quad \tilde{\varrho}(t)=o(\widetilde{\Lambda}(t)),
$$

then any solution $z(t)$ of (2) existing for $t \rightarrow \infty$ satisfies

$$
\lim _{t \rightarrow \infty} z(t)=0 .
$$

Proof. Choose arbitrary $\varepsilon>0$. According to (47), there is $s \geq$ $T^{*}$ such that $\widetilde{\varrho}(t) \leq(1 / 2) \mu \varepsilon|\widetilde{\Lambda}(t)|$ for $t \geq s$ and

$$
\begin{aligned}
\int_{s}^{t} \tilde{\varrho}(\tau) \exp \left(\int_{\tau}^{t} \widetilde{\Lambda}(\sigma) d \sigma\right) d \tau \\
\leq \frac{\mu \varepsilon}{2} \int_{s}^{t}[-\widetilde{\Lambda}(\tau)] \exp \left(\int_{\tau}^{t} \widetilde{\Lambda}(\sigma) d \sigma\right) d \tau \\
=\frac{\mu \varepsilon}{2} \int_{s}^{t}\left(\frac{d}{d \tau}\left[\exp \left(\int_{\tau}^{t} \widetilde{\Lambda}(\sigma) d \sigma\right)\right]\right) d \tau \\
=\frac{\mu \varepsilon}{2}\left[\exp \left(\int_{\tau}^{t} \widetilde{\Lambda}(\sigma) d \sigma\right)\right]_{s}^{t} \\
=\frac{\mu \varepsilon}{2}\left[1-\exp \left(\int_{s}^{t} \widetilde{\Lambda}(\tau) d \tau\right)\right]<\frac{\mu \varepsilon}{2},
\end{aligned}
$$

for $t \geq s$. From (47) we have $\exp \left(\int_{s}^{t} \widetilde{\Lambda}(\tau) d \tau\right) \rightarrow 0$ as $t \rightarrow \infty$; hence, there is $S \geq s$ such that $\exp \left(\int_{s}^{t} \widetilde{\Lambda}(\tau) d \tau\right)<\mu \varepsilon(2 V(s))^{-1}$ for $t \geq S$. Considering this fact and (41), we get

$$
\mu|z(t)|<V(s) \frac{\mu \varepsilon}{2 V(s)}+\frac{\mu \varepsilon}{2}=\mu \varepsilon,
$$

for $t \geq S$. This completes the proof.
Remark 7. Theorem 6 is a generalization of results published in the papers $[16,18,20]$.

If we take $A_{1}(t)=A(t), A_{k} \equiv 0$, for $k=2, \ldots, m, B_{1}(t)=$ $B(t), B_{k} \equiv 0$, for $k=2, \ldots, m$, and $\theta_{1}(t)=t-r$, where $r>0$, we get Theorem 3 from [16].

If we take $\theta_{k}(t)=t-r_{k}$, where $r_{k}>0, k=1, \ldots, m$, we obtain Theorem 3 from [18].

If we take $A_{1}(t)=A(t), A_{k} \equiv 0$, for $k=2, \ldots, m$, $B_{1}(t)=B(t), B_{k} \equiv 0$, for $k=2, \ldots, m$, and $\theta_{1}(t)=\theta(t)$, we get Theorem 2.14 from [20].

\section{Corollaries and Examples}

From Theorem 2 we easily obtain several corollaries. We give an example which shows that it is worth to consider the case (5).

Corollary 8. Let $a(t) \equiv a \in \mathbb{C}, b(t) \equiv b \in \mathbb{C},|\operatorname{Im} a|>|b|$. Suppose that $\lim _{t \rightarrow \infty} \theta_{k}(t)=\infty, \theta_{k}(t) \leq t$, for $t \geq T_{1}$, where $T_{1} \geq t_{0}$. Let $\rho_{0}, \rho_{1}, \ldots, \rho_{m}:\left[T_{1}, \infty\right) \rightarrow \mathbb{R}$ be such that

$$
\left|g\left(t, z, w_{1}, \ldots, w_{m}\right)\right| \leq \rho_{0}(t)|z|+\sum_{k=1}^{m} \rho_{k}(t)\left|w_{k}\right|
$$

for $t \geq T_{1},|z|<R,\left|w_{k}\right|<R, k=1, \ldots, m, R>0$ and $\rho_{0} \in$ $L_{\text {loc }}\left(\left[T_{1}, \infty\right), \mathbb{R}\right)$.

$$
\text { Let } \widetilde{\beta} \in A C_{\mathrm{loc}}\left(\left[T_{1}, \infty\right), \mathbb{R}_{+}\right) \text {satisfy }
$$

$$
\begin{aligned}
& \theta_{k}^{\prime}(t) \tilde{\beta}(t) \\
& \quad \geq\left(\frac{|\operatorname{Im} a|+|b|}{|\operatorname{Im} a|-|b|}\right)^{1 / 2}\left(\rho_{k}(t)+\left|A_{k}(t)\right|+\left|B_{k}(t)\right|\right),
\end{aligned}
$$

a.e. on $\left[T_{1}, \infty\right)$ for $k=1, \ldots, m$. If

$$
\begin{array}{r}
\limsup _{t \rightarrow \infty} \int^{t} \max \left(\operatorname{Re} a+\left(\frac{|\operatorname{Im} a|+|b|}{|\operatorname{Im} a|-|b|}\right)^{1 / 2} \rho_{0}(s)\right. \\
\left.+m \widetilde{\beta}(s), \frac{\widetilde{\beta}^{\prime}(s)}{\widetilde{\beta}(s)}\right) d s<\infty,
\end{array}
$$

then the trivial solution of (2) is stable. If

$$
\begin{array}{r}
\lim _{t \rightarrow \infty} \int^{t} \max \left(\operatorname{Re} a+\left(\frac{|\operatorname{Im} a|+|b|}{|\operatorname{Im} a|-|b|}\right)^{1 / 2} \rho_{0}(s)\right. \\
\left.+m \tilde{\beta}(s), \frac{\widetilde{\beta}^{\prime}(s)}{\widetilde{\beta}(s)}\right) d s=-\infty,
\end{array}
$$

then the trivial solution of (2) is asymptotically stable.

Proof. Choose $T \geq T_{1}$ such that $\theta_{k}(t) \geq T_{1}$ for $t \geq T, k=$ $1, \ldots, m$. Denote that $z=z(t)$ and $w_{k}=z\left(\theta_{k}(t)\right)$ again. Since 
$a, b \in \mathbb{C}$ are constants, then also $\tilde{\gamma}$ and $\tilde{c}$ are constants, and we have $\widetilde{\vartheta}(t) \equiv 0$. Using condition (51) we get

$$
\begin{aligned}
& \left|\widetilde{\gamma} g\left(t, z, w_{1}, \ldots, w_{m}\right)+\widetilde{c} g\left(t, z, w_{1}, \ldots, w_{m}\right)\right| \\
& \quad \leq(|\widetilde{\gamma}|+|\widetilde{c}|)\left(\rho_{0}(t)|z|+\sum_{k=1}^{m} \rho_{k}(t)\left|w_{k}\right|\right) \\
& \quad=\frac{|\widetilde{\gamma}|+|\widetilde{c}|}{|\widetilde{\gamma}|-|\widetilde{c}|}(|\widetilde{\gamma}|-|\widetilde{c}|)\left(\rho_{0}(t)|z|+\sum_{k=1}^{m} \rho_{k}(t)\left|w_{k}\right|\right) \\
& \quad \leq \frac{|\widetilde{\gamma}|+|\widetilde{c}|}{|\widetilde{\gamma}|-|\widetilde{c}|}\left(\rho_{0}(t)|\tilde{\gamma} z+\widetilde{c} z|+\sum_{k=1}^{m} \rho_{k}(t)\left|\widetilde{\gamma} w_{k}+\widetilde{c}_{k}\right|\right),
\end{aligned}
$$

and it follows that condition (ii) holds with

$$
\tilde{\varkappa}(t)=\frac{|\tilde{\gamma}|+|\widetilde{c}|}{|\widetilde{\gamma}|-|\widetilde{c}|} \rho_{0}(t), \quad \widetilde{\kappa}_{k}(t)=\frac{|\widetilde{\gamma}|+|\widetilde{c}|}{|\widetilde{\gamma}|-|\widetilde{c}|} \rho_{k}(t)
$$

and $\widetilde{\varrho}(t) \equiv 0$.

Condition (53) implies that $\operatorname{Re} a \leq 0$. Since

$$
\begin{aligned}
\frac{|\tilde{\gamma}|+|\widetilde{c}|}{|\tilde{\gamma}|-|\widetilde{c}|} & =\frac{|\operatorname{Im} a|+\sqrt{|\operatorname{Im} a|^{2}-|b|^{2}}+|b|}{|\operatorname{Im} a|+\sqrt{|\operatorname{Im} a|^{2}-|b|^{2}}-|b|} \\
& =\left(\frac{|\operatorname{Im} a|+|b|}{|\operatorname{Im} a|-|b|}\right)^{1 / 2},
\end{aligned}
$$

in view of (14) we obtain

$$
\begin{aligned}
\tilde{\lambda}_{k}(t)= & \left(\frac{|\operatorname{Im} a|+|b|}{|\operatorname{Im} a|-|b|}\right)^{1 / 2} \\
& \times\left\{\rho_{k}(t)+\left|A_{k}(t)\right|+\left|B_{k}(t)\right|\right\}, \\
\widetilde{\Theta}(t)= & \operatorname{Re} a+\frac{|\widetilde{\gamma}|+|\widetilde{c}|}{|\widetilde{\gamma}|-|\widetilde{c}|} \rho_{0}(t)+m \widetilde{\beta}(t) \\
= & \operatorname{Re} a+\left(\frac{|\operatorname{Im} a|+|b|}{|\operatorname{Im} a|-|b|}\right)^{1 / 2} \rho_{0}(t)+m \widetilde{\beta}(t),
\end{aligned}
$$

and the assertion follows from (16) and Theorem 2.

Now we show an example that, under certain circumstances, Corollary 8 is more useful than Corollary 1 from [21].

Example 9. Consider (2), where $a(t) \equiv-\sqrt{5}+2 i, b(t) \equiv 1$, $A_{k}(t) \equiv 0, B_{k}(t) \equiv 0$, for $k=1, \ldots, m$, and

$$
g\left(t, z, w_{1}, \ldots, w_{m}\right)=\frac{2}{\sqrt{3}} e^{i t} z+\sum_{k=1}^{m} \frac{k}{2 m t}(\sqrt{15}-\sqrt{14}) e^{-t} w_{k}
$$

Assume that $t_{0}=m$ and $R=\infty, \theta_{k}(t)=k \ln t$. Put $T=$ $e^{t_{0}}=e^{m}$. Then, $\rho_{0}(t) \equiv 2 / \sqrt{3}, \rho_{k}(t)=(k / 2 m t)(\sqrt{15}-\sqrt{14}) e^{-t}$. We have

$$
\begin{aligned}
& \max \left(\frac{|a|-|b|}{|a|} \operatorname{Re} a+\left(\frac{|a|+|b|}{|a|-|b|}\right)^{1 / 2} \rho_{0}(t)+m \beta(t), \frac{\beta^{\prime}(t)}{\beta(t)}\right) \\
& \quad=\max \left(-\frac{2}{3} \sqrt{5}+\sqrt{2} \frac{2}{\sqrt{3}}+m \beta(t), \frac{\beta^{\prime}(t)}{\beta(t)}\right) \\
& \quad \geq \frac{2}{3}(\sqrt{6}-\sqrt{5})>0,
\end{aligned}
$$

for

$$
\begin{aligned}
\theta_{k}^{\prime}(t) \beta(t)= & \frac{k}{t} \beta(t) \geq\left(\frac{|a|+|b|}{|a|-|b|}\right)^{1 / 2} \\
& \times\left\{\rho_{k}(t)+\left|A_{k}(t)\right|+\left|B_{k}(t)\right|\right\} \\
= & \frac{k}{m t \sqrt{2}}(\sqrt{15}-\sqrt{14}) e^{-t},
\end{aligned}
$$

where $k \in\{1, \ldots, m\}$; hence, we cannot apply Corollary 1 from [21].

On the other hand, if we use

$$
\begin{aligned}
\theta_{k}^{\prime}(t) \widetilde{\beta}(t) & =\frac{k}{t} \widetilde{\beta}(t)=\frac{k \sqrt{3}}{2 m t}(\sqrt{15}-\sqrt{14}) e^{-t} \\
& \geq\left(\frac{|\operatorname{Im} a|+|b|}{|\operatorname{Im} a|-|b|}\right)^{1 / 2}\left\{\rho_{k}(t)+\left|A_{k}(t)\right|+\left|B_{k}(t)\right|\right\},
\end{aligned}
$$

where $k \in\{1, \ldots, m\}$, we have

$$
\begin{aligned}
& \max \left(\operatorname{Re} a+\left(\frac{|\operatorname{Im} a|+|b|}{|\operatorname{Im} a|-|b|}\right)^{1 / 2} \rho_{0}(t)+m \tilde{\beta}(t), \frac{\widetilde{\beta}^{\prime}(t)}{\widetilde{\beta}(t)}\right) \\
& \quad=\max \left(-\sqrt{5}+2+m \frac{\sqrt{3}}{2 m}(\sqrt{15}-\sqrt{14}) e^{-t},-1\right) \\
& \quad \leq-\sqrt{5}+2+\frac{\sqrt{3}}{2}(\sqrt{15}-\sqrt{14})<-\frac{12}{100}<0 ;
\end{aligned}
$$

thus, Corollary 8 guarantees the stability and also asymptotic stability of the trivial solution of the considered equation.

The following corollary gives sufficient conditions for stability of the trivial solution of (2).

Corollary 10. Assume that the conditions (i), (ii), and (iii) are valid with $\widetilde{\varrho}(t) \equiv 0$. If $\widetilde{\beta}(t)$ is monotone and bounded on $[T, \infty)$ and if

$$
\limsup _{t \rightarrow \infty} \int^{t}[\widetilde{\Theta}(s)]_{+} d s<\infty,
$$

where $[\widetilde{\Theta}(t)]_{+}=\max \{\widetilde{\Theta}(t), 0\}$, then the trivial solution of $(2)$ is stable. 
Proof. Suppose firstly that $\widetilde{\beta}$ is nonincreasing on $[T, \infty)$. Then, $\widetilde{\beta}^{\prime} \leq 0$ a.e. on $[T, \infty)$.

If $\widetilde{\beta}\left(T_{2}\right)=0$ for some $T_{2} \geq T$, then $\widetilde{\beta}(t) \equiv 0$ on $\left[T_{2}, \infty\right)$. Consequently, $\widetilde{\Lambda}$ has to satisfy only the inequality $\widetilde{\Theta}(t) \leq \widetilde{\Lambda}(t)$ a.e. on $\left[T_{2}, \infty\right)$, so we may choose $\widetilde{\Lambda}(t)=\widetilde{\Theta}(t)$ on $\left[T_{2}, \infty\right)$. It follows that $\widetilde{\Lambda}(t)=\widetilde{\Theta}(t) \leq \max \{\widetilde{\Theta}(t), 0\}=[\widetilde{\Theta}(t)]_{+}$.

On the other hand, if $\widetilde{\beta}(t)>0$ on $[T, \infty)$, we may put $\widetilde{\Lambda}(t)=\max \left\{\widetilde{\Theta}(t), \widetilde{\beta}^{\prime}(t) / \widetilde{\beta}(t)\right\}$. Then,

$$
\widetilde{\Lambda}(t)=\max \left\{\widetilde{\Theta}(t), \frac{\widetilde{\beta}^{\prime}(t)}{\widetilde{\beta}(t)}\right\} \leq \max \{\widetilde{\Theta}(t), 0\}=[\widetilde{\Theta}(t)]_{+} .
$$

In both cases, $\widetilde{\Lambda}$ satisfies condition (iv) and the inequality $\widetilde{\Lambda}(t) \leq[\widetilde{\Theta}(t)]_{+}$on $\left[T_{2}, \infty\right)$; hence,

$$
\limsup _{t \rightarrow \infty} \int^{t} \widetilde{\Lambda}(s) d s \leq \limsup _{t \rightarrow \infty} \int^{t}[\widetilde{\Theta}(s)]_{+} d s<\infty .
$$

Now assume that $\widetilde{\beta}$ is nondecreasing on $[T, \infty)$. Then, $\widetilde{\beta}^{\prime} \geq 0$ a.e. on $[T, \infty)$.

If $\widetilde{\beta}(t) \equiv 0$ on $[T, \infty)$, we may treat it as previously mentioned.

Otherwise, there is some $T_{3} \geq T$ such that $\widetilde{\beta}(t)>0$ on $\left[T_{3}, \infty\right)$, and we may choose $\widetilde{\Lambda}(t)=\max \left\{\widetilde{\Theta}(t), \widetilde{\beta}^{\prime}(t) / \widetilde{\beta}(t)\right\}$ on $\left[T_{3}, \infty\right)$. Clearly $\widetilde{\Lambda}$ satisfies condition (iv) on $\left[T_{3}, \infty\right)$. Since $\widetilde{\beta}^{\prime} \geq 0$ a.e. on $[T, \infty)$, it follows that $\widetilde{\beta}^{\prime} / \widetilde{\beta} \geq 0$ a.e. on $\left[T_{3}, \infty\right)$. Hence,

$$
\begin{aligned}
\widetilde{\Lambda}(t) & =\max \left\{\widetilde{\Theta}(t), \frac{\widetilde{\beta}^{\prime}(t)}{\widetilde{\beta}(t)}\right\} \\
& \leq \max \left\{[\widetilde{\Theta}(t)]_{+}, \frac{\widetilde{\beta}^{\prime}(t)}{\widetilde{\beta}(t)}\right\} \\
& \leq[\widetilde{\Theta}(t)]_{+}+\frac{\widetilde{\beta}^{\prime}(t)}{\widetilde{\beta}(t)},
\end{aligned}
$$

and then

$$
\begin{aligned}
& \limsup _{t \rightarrow \infty} \int^{t} \widetilde{\Lambda}(s) d s \\
& \leq \limsup _{t \rightarrow \infty} \int^{t}[\widetilde{\Theta}(s)]_{+} d s+\limsup _{t \rightarrow \infty} \int^{t} \frac{\widetilde{\beta}^{\prime}(t)}{\widetilde{\beta}(t)} d s \\
& \leq \limsup _{t \rightarrow \infty} \int^{t}[\widetilde{\Theta}(s)]_{+} d s+\limsup _{t \rightarrow \infty}(\ln (\widetilde{\beta}(t))) \\
& \quad-\ln \left(\widetilde{\beta}\left(T_{3}\right)\right)<\infty,
\end{aligned}
$$

since $\widetilde{\beta}$ is bounded on $[T, \infty)$.

The statement follows from Theorem 2.

We can derive several consequences from Theorem 4.
Corollary 11. Let the conditions (i), (ii), (iii), and (iv) be fulfilled and

$$
\limsup _{t \rightarrow \infty} \int_{s}^{t} \widetilde{\varrho}(\xi) \exp \left(-\int_{s}^{\xi} \widetilde{\Lambda}(\sigma) d \sigma\right) d \xi<\infty,
$$

for some $s \geq T$.

If $z(t)$ is any solution of (2) existing for $t \rightarrow \infty$, then

$$
z(t)=O\left[\exp \left(\int_{s}^{t} \widetilde{\Lambda}(\xi) d \xi\right)\right] .
$$

Proof. From the assumptions and (45) we can see that there are $K>0$ and $S \geq s$ such that for $t \geq S$ we have

$$
\begin{aligned}
V(t) & \exp \left(-\int_{s}^{t} \widetilde{\Lambda}(\xi) d \xi\right)-V(s) \\
& \leq \int_{s}^{t} \widetilde{\varrho}(\xi) \exp \left(-\int_{s}^{\xi} \widetilde{\Lambda}(\sigma) d \sigma\right) d \xi \\
& \leq K<\infty .
\end{aligned}
$$

Then,

$$
\mu|z(t)| \leq V(t) \leq(K+V(s)) \exp \left(\int_{s}^{t} \widetilde{\Lambda}(\xi) d \xi\right) .
$$

Corollary 12. Let the assumptions (i), (ii), (iii), and (iv) hold, and let

$$
\limsup _{t \rightarrow \infty} \widetilde{\Lambda}(t)<\infty, \quad \widetilde{\varrho}(t)=O\left(e^{\eta t}\right),
$$

where $\eta>\lim \sup _{t \rightarrow \infty} \widetilde{\Lambda}(t)$. If $z(t)$ is any solution of (2) existing for $t \rightarrow \infty$, then $z(t)=O\left(e^{\eta t}\right)$.

Proof. In view of (73), there are $L>0, \eta^{*}<\eta$, and $s>T$ such that $\eta^{*}>\widetilde{\Lambda}(t)$ for $t \geq s$ and $\widetilde{\varrho}(t) e^{-\eta t}<L$ for $t \geq s$. From (41) we get

$$
\begin{aligned}
\mu|z(t)| & \leq V(s) e^{\eta^{*}(t-s)}+L \int_{s}^{t} e^{\eta \tau} e^{\eta^{*}(t-\tau)} d \tau \\
& \leq V(s) e^{\eta^{*}(t-s)}+L e^{\eta^{*} t} \frac{e^{\left(\eta-\eta^{*}\right) t}-e^{\left(\eta-\eta^{*}\right) s}}{\eta-\eta^{*}} \\
& \leq V(s) e^{\eta^{*}(t-s)}+\frac{L}{\eta-\eta^{*}} e^{\eta t}=O\left(e^{\eta t}\right) .
\end{aligned}
$$

Remark 13. If $\tilde{\rho}(t) \equiv 0$, we can take $L=0$ in the proof of Corollary 12, and taking inequalities (74) into account we obtain the following statement: there is an $\eta^{*}<\eta_{0}<\eta$ such that $z(t)=o\left(e^{\eta_{0} t}\right)$ holds for the solution $z(t)$ of $(2)$.

\section{Conclusion}

We studied asymptotic behavior of real two-dimensional differential system with a finite number of nonconstant delays. We considered the case corresponding to the situation 
when the equilibrium point 0 of autonomous system (6) is a stable focus or a stable centre. We utilized the method of complexification and the method of Lyapunov-Krasovskii functional. Criteria for stability and asymptotic stability of the solutions as well as conditions ensuring that all solutions of (2) tend to zero are derived. At the end we supplied several corollaries and an example which shows that in some cases the criteria obtained in this paper are more applicable than the criteria presented in [21].

\section{Acknowledgments}

The first author was supported by the Project CZ.1.07/2.3.00/ 30.0039 of Brno University of Technology. The work of the second author was realised in CEITEC-Central European Institute of Technology-with research infrastructure supported by the Project CZ.1.05/1.1.00/02.0068 financed from European Regional Development Fund and by the Project FEKT-S-11-2-921 of Faculty of Electrical Engineering and Communication, Brno University of Technology. This support is gratefully acknowledged.

\section{References}

[1] J. Baštinec, L. Berezansky, J. Diblík, and Z. Šmarda, "On the critical case in oscillation for differential equations with a single delay and with several delays," Abstract and Applied Analysis, vol. 2010, Article ID 417869, 20 pages, 2010.

[2] J. Čermák, "On matrix differential equations with several unbounded delays," European Journal of Applied Mathematics, vol. 17, no. 4, pp. 417-433, 2006.

[3] J. Čermák and S. Dvořáková, "Asymptotic estimation for some nonlinear delay differential equations," Results in Mathematics, vol. 51, no. 3-4, pp. 201-213, 2008.

[4] J. Diblík, "Asymptotic representation of solutions of equation $\dot{y}(t)=\beta(t)[y(t)-y(t-\tau(t))]$," Journal of Mathematical Analysis and Applications, vol. 217, no. 1, pp. 200-215, 1998.

[5] J. Diblík, "A criterion for existence of positive solutions of systems of retarded functional differential equations," Nonlinear Analysis, Theory, Methods and Applications, vol. 38, no. 3, pp. 327-339, 1999.

[6] J. Diblík and M. Růžičková, "Exponential solutions of equation $\dot{y}(t)=\beta(t)[y(t-\delta)-y(t-\tau)]$," Journal of Mathematical Analysis and Applications, vol. 294, no. 1, pp. 273-287, 2004.

[7] J. Diblík and Z. Svoboda, "Positive solutions of retarded functional differential equations," Nonlinear Analysis, Theory, Methods and Applications, vol. 63, no. 5-7, pp. e813-e821, 2005.

[8] J. Diblík and Z. Svoboda, "Positive solutions of $p$-type retarded functional differential equations," Nonlinear Analysis, Theory, Methods and Applications, vol. 64, no. 8, pp. 1831-1848, 2006.

[9] J. Diblík, Z. Svoboda, and Z. Šmarda, "Explicit criteria for the existence of positive solutions for a scalar differential equation with variable delay in the critical case," Computers and Mathematics with Applications, vol. 56, no. 2, pp. 556-564, 2008.

[10] J. Diblík, Z. Svoboda, and Z. Šmarda, "Retract principle for neutral functional differential equations," Nonlinear Analysis, Theory, Methods and Applications, vol. 71, no. 12, pp. e1393-e1400, 2009.
[11] I. Györi and M. Pituk, "Stability criteria for linear delay differential equations," Differential Integral Equations, vol. 10, no. 5, pp. 841-852, 1997.

[12] M. Pituk, "Asymptotic behavior and oscillation of functional differential equations," Journal of Mathematical Analysis and Applications, vol. 322, no. 2, pp. 1140-1158, 2006.

[13] E. Špániková and H. Šamajová, "Asymptotic properties of solutions to n-dimensional neutral differential systems," Nonlinear Analysis, Theory, Methods and Applications, vol. 71, no. 7-8, pp. 2877-2885, 2009.

[14] S. Yi, P. W. Nelson, and A. G. Ulsoy, "Delay differential equations via the matrix lambert $\mathrm{W}$ function and bifurcation analysis: application to machine tool chatter," Mathematical Biosciences and Engineering, vol. 4, no. 2, pp. 355-368, 2007.

[15] M. Ráb and J. Kalas, "Stability of dynamical systems in the plane," Differential Integral Equations, vol. 3, no. 1, pp. 127-144, 1990.

[16] J. Kalas and L. Baráková, "Stability and asymptotic behaviour of a two-dimensional differential system with delay," Journal of Mathematical Analysis and Applications, vol. 269, no. 1, pp. 278300, 2002.

[17] J. Rebenda, "Asymptotic properties of solutions of real twodimensional differential systems with a finite number of constant delays," Memoirs on Differential Equations and Mathematical Physics, vol. 41, pp. 97-114, 2007.

[18] J. Rebenda, "Asymptotic behaviour of real two-dimensional differential system with a finite number of constant delays," Demonstratio Mathematica, vol. 41, no. 4, pp. 845-857, 2008.

[19] J. Kalas, "Asymptotic behaviour of a two-dimensional differential system with nonconstant delay," Mathematische Nachrichten, vol. 283, no. 6, pp. 879-890, 2010.

[20] J. Rebenda, "Asymptotic behaviour of solutions of real twodimensional differential system with nonconstant delay," Archivum Mathematicum, vol. 45, no. 3, pp. 223-236, 2009.

[21] J. Rebenda and Z. Šmarda, "Stability and asymptotic properties of a system of functional differential equations with nonconstant delays," Applied Mathematics and Computation, vol. 219, no. 12, pp. 6622-6632, 2013.

[22] S. Saks, Theory of the integral, Monografie Matematyczne, Tom 7, Warszawa-Lwów, 1937.

[23] V. Jarník, Differential Calculus II, NČSAV Praha, 1956, in Czech. 


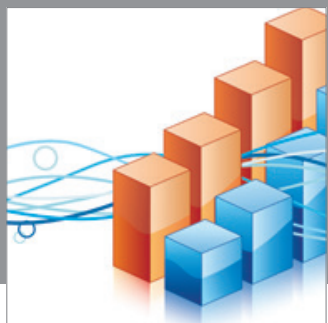

Advances in

Operations Research

mansans

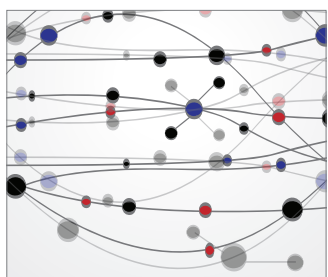

The Scientific World Journal
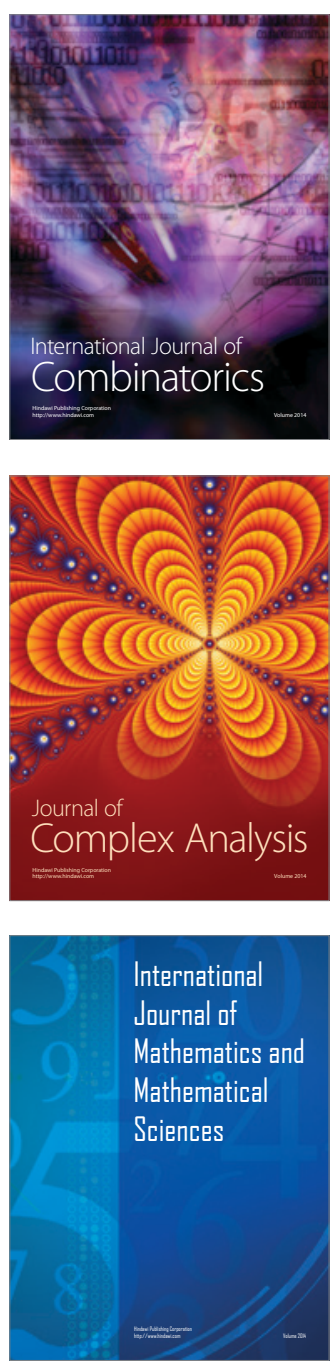
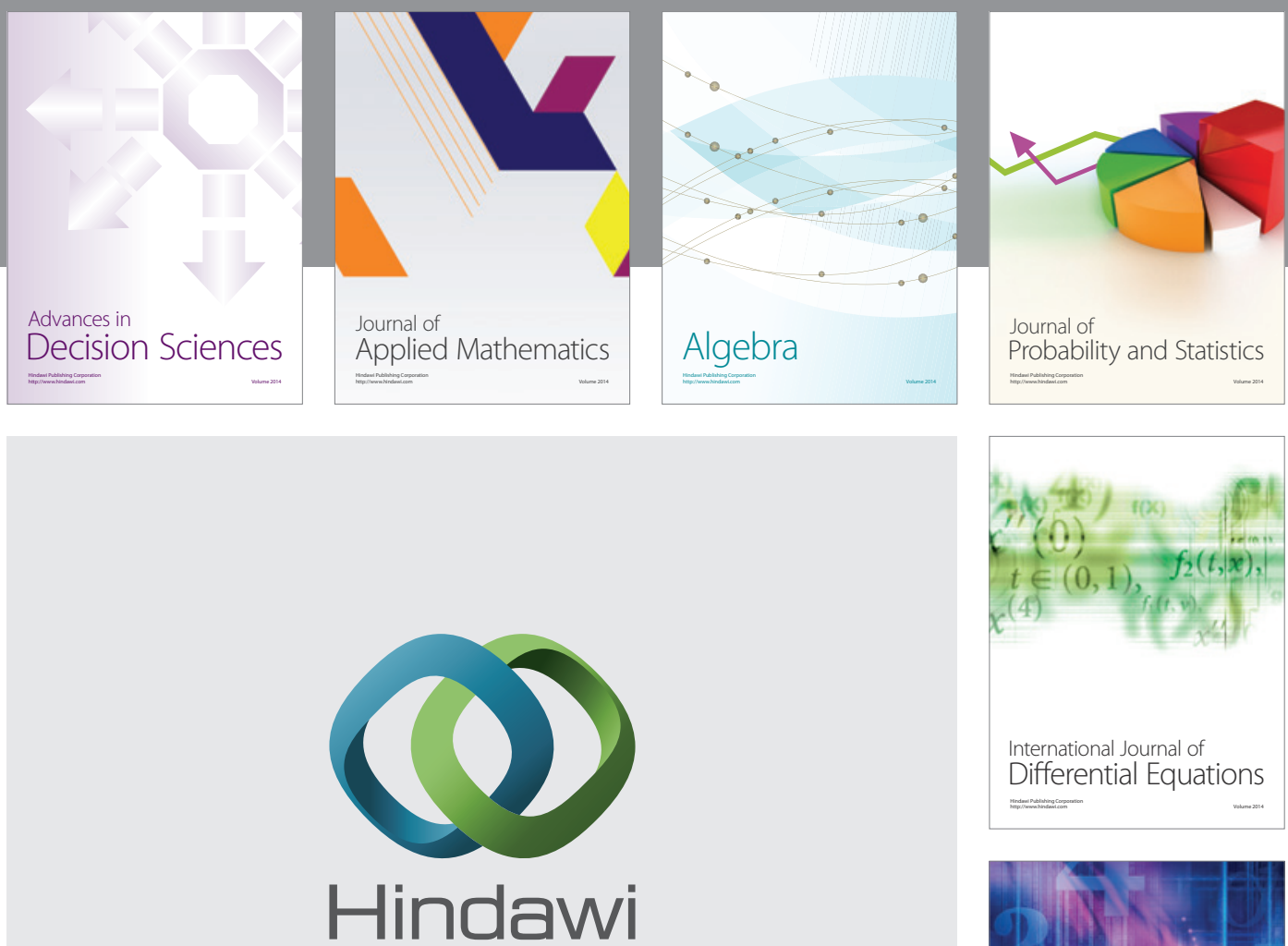

Submit your manuscripts at http://www.hindawi.com
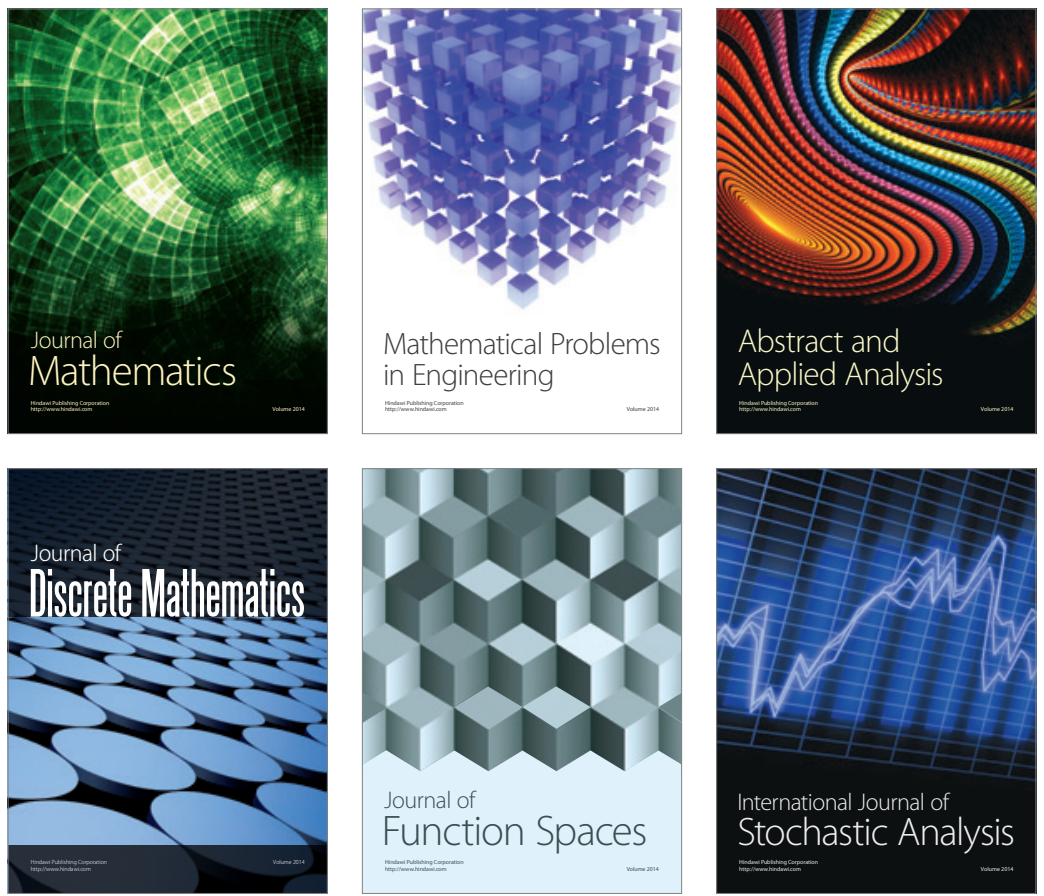

Journal of

Function Spaces

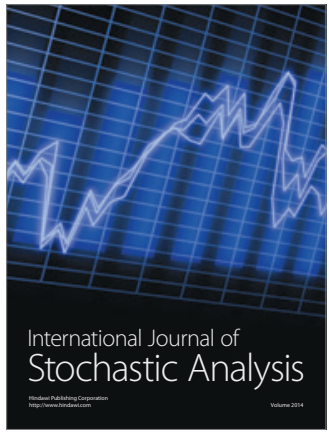

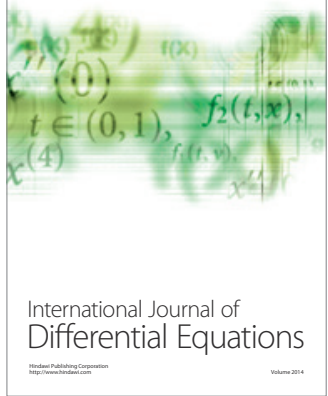
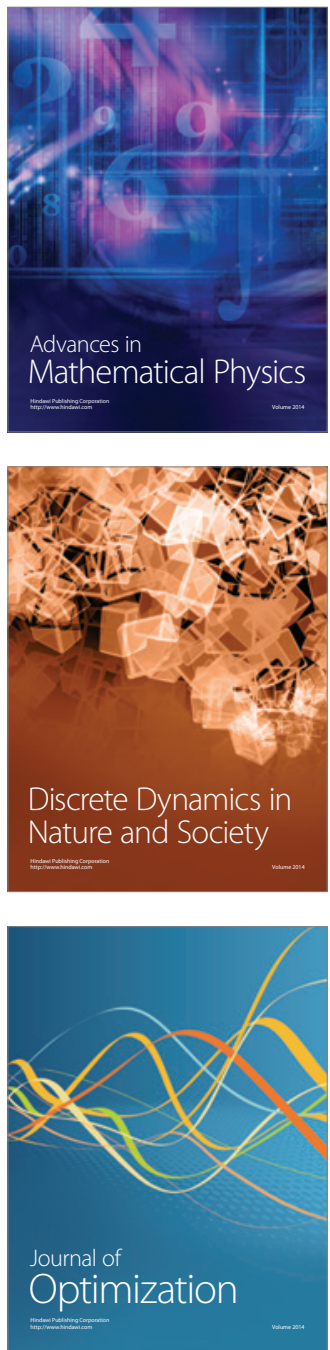